\title{
Clinical trans-omics: an integration of clinical phenomes with molecular multiomics
}

\author{
Xiangdong Wang
}

Received: 16 April 2018 / Accepted: 16 April 2018 / Published online: 24 April 2018

(C) Springer Science+Business Media B.V., part of Springer Nature 2018

Clinical trans-omics is a new emerging scientific discipline that integrates clinical phenomes with molecular multiomics, in order to further understand molecular mechanisms of disease pathogenesis and progression, patient sensitivity to therapy and prognosis, and therapy design and development. The importance of clinical trans-omics lies in its possible production of a new and novel therapeutic strategy for diseases which can be designed based on altered omics profiles, improve the understanding of patient signs and symptoms in multiple dimensions, and lead us into the era of molecular medications. The most critical and challenging factor in the application of clinical transomics is to have automatic learning systems, which can dynamically generate, collect, integrate, and analyze large-scale data of multiomics. A large number of challenges, however, still need to be overcome. The "trans" is a key word that indicates the gathering and/ or spanning of various layers of omics in different forms, which may sometimes be completely incomparable to each other. For example, the expression of genomic and proteomic profiles can be presented as the density or rationales a digital values, while most of the clinical phenotypes are recorded as worddescriptive or image-based information. There is increasing evidence to show the potential of this

X. Wang $(\bowtie)$

Zhongshan Hospital Institute of Clinical Science, Fudan University Shanghai Medical College; Shanghai Institute of Clinical Bioinformatics, Shanghai, China

e-mail: xiangdong.wang@ clintransmed.org approach as more tools and methodologies are discovered and developed to build up the correlation and network of molecular multiomics with clinical phenotypes, identify disease-specific individual phenomes by patient trait prediction using whole-genome sequencing data, and indicate genetic alterations by gene profiling prediction using patient phenomics. The current application of clinical trans-omics is focused on the discovery and development of disease-specific diagnostic biomarkers and therapeutic targets. Clinical trans-omics will furthermore be applied for the design and decision-making regarding therapeutic strategies for individuals and for the prediction of patient prognosis and burden of social medical care. It is time to define and translate the concept of clinical trans-omics into clinical practice to benefit patients, especially those with serious, rare, or complex diseases.

\section{Precise discovery of diagnostic biomarkers}

Multiomics is defined as a biological analysis approach where more than two omics measurements are performed simultaneously in one cell, organ/tissue, or body. The nomenclature with "omics" suffix, e.g., genomics, proteomics, metabolomics, transcriptomics, lipidomics, immunomics, glycomics, or RNomics, is growing with the rapid development of techniques. The development of human diseases is highly dependent upon genetic and environmental changes. For example, chronic lung diseases occur in patients with susceptible genetic backgrounds and worsen from 
airway inflammation and chronic obstructive pulmonary diseases to lung cancer, after repeated provocations of infection and pollution (Wang, 2016). There is an example of studying the interaction and influence between bacteria and their environmental elements, by integrating trans-omics from bacterial transcriptomic and fluxomic layers with networks of environmental phenotypes and by focusing multiomics layers into one network with comparison to the global network of environmental elements (Angione et al., 2016). This indicates that it is possible to merge and integrate omics data from molecular networks, phenome networks, or networks of any other factors, although this is still far away from clinical practice. There is a definite gap of knowledge in multiomics disease-specific biomarkers and a solid request to bring clinical phenomes into account (Chen et al., 2016; Kawamura et al., 2018; Wang et al., 2017).

\section{Identification of therapeutic targets}

Therapeutic targets can be molecules or subcellular organelles that are mainly dependent upon critical and specific biological functions (Wang \& Wang, 2017; Wu et al., 2018). Multiomics can play a unique and important role in the identification of therapeutic targets in clinical trials, while the role in target validation should be further clarified. Based on the principle of trans-omics, (Konno et al., 2018) identified therapeutic targets for gastrointestinal cancer by measuring methylomics and transcriptomics in the ornithine decarboxylase degron-positive cancer stem cell model of gastrointestinal tumor in response to drug-based therapies. In addition to standard analyses of gene expression and DNA methylation, there is a urgent need to have a new strategy to analyze trans-omics data by calculating the variation between transcription and DNA methylation during and after the treatment and correlating core genes selected from those significant changes in both methylation and expression. Such a protocol of preclinical transomics can be considered as a reference to identify targeting molecules, which can control and regulate the sensitivity and/or resistance of patients. In particular, clinical trans-omics is likely to be more and more important for discovering mechanisms and targets during the development of target-based drug resistance or toxicity in cancer therapies. The specificity of therapeutic targets or targets responsible for sensitivity or resistance to drugs selected from multiomics is dependent upon multiomics characteristics and selection, efficient elimination of noises and false-positive targets, individualized dynamic networks, and regulatory enhancers and suppressors.

\section{Analytic methodologies of trans-omics data}

One of the most difficult challenges is to develop precise analytic methodologies and standardize the deep mining processes, in order to meet clinical requirements of outcome quality. In preclinical research, multiple immature analyses can be explored and applied to cell- and animal model-based studies as these are forbidden in clinical practice. One of the milestone studies developed a new method for analysis of individual biomarker dynamic networks to define the common and specific network characteristics for individual patients (Yu et al., 2015). It was coined as a personalized dysfunctional gene network by simultaneously integrating genes with different features, e.g., the differential gene expression, expression variance, and differential expression covariance. In contrast from networks constructed from genes of group patients, the individual networks of genes or proteins have a new bi-colored topological structure, can identify genes or gene pairs with innovative power, and measure the expression state or activity of different feature genes and their network or modules in one sample for an individual. Sun and $\mathrm{Hu}$ (Sun \& $\mathrm{Hu}$, 2016) overviewed and emphasized the importance of integrative analysis of multiomics data in the design, discovery, and functional studies of complex human diseases. They provide valuable recommendations on analytical methodologies for different types of human multiomics data and study designs. Human diseases are more complex than we understood or expected. A large number of methods, together with a real-time improvement and update, are required to trace the core information spanning among multiomics layers.

\section{The importance of clinical phenomes}

The most important issue for clinical trans-omics in the discovery of disease-specific biomarkers is to engineer a comparable system or model and integrate molecular multiomics with clinical phenomes. We have tried to integrate core gene networks of genomics in patients with acute exacerbation of chronic obstructive 
pulmonary disease with clinical phenomes, e.g., patient signs and symptoms, biomedical analyses, pathology, images, and responses to therapies, as compared with healthy individuals, long-term smokers, or individuals at a stable stage of chronic obstructive pulmonary disease or lung cancer. (Chen et al., 2012a; Chen et al., 2012b; Shi et al., 2018; Wu et al., 2014). The most challenging process is to translate clinical descriptive phenomes into digital values using the designed digital score evaluation system, in order to make the network of clinical phenomes comparable with molecular multiomics. After trans-omics analyses, disease stage-, severity-, duration-, phenome-specific biomarkers can be selected and validated in biological systems. Such pioneering studies on identification and validation of disease-specific biomarkers create new potential for application of clinical trans-omics, although the methodology of integration and analyses of multiomics need to be further improved. Srivastava et al. (Srivastava et al., 2018) provide strong evidence to support the reality of clinical trans-omics used to integrate clinical phenomes with molecular multiomics, by linking histopathological image phenomes with transcriptome and proteome data as predictive models using three different modalities. This study particularly focused on disease stages, estrogen receptor status, pathological subtypes, and convolutional neural network-based classifiers as part of the clinical phenomes on which the model was built up and compared among all data modalities. It is challenging that the number and design of cases in global databases are limited and have their own origin objective. Thus, more prospective and well-designed studies on clinical multiomics are highly desirable.

\section{Trans-omics and clinical phenomes}

The other challenging issue is to understand precise correlations between patient phenomes and transomics using improvements of omics measurements and analyses. The ideal molecular medication will define and predict patient phenomes based on transomics-multiomics data or gene/protein profiles from clinical phenotypes. One of the revolutionary studies on phenome-omics transit identified individuals and individual phenomes by trait prediction using genomic data like whole-genome sequencing data (Lippert et al., 2017). They performed a large cohort study and predicted individual traits by integrating whole-genome sequencing, detailed phenotyping, and statistical modeling and developed the maximum entropy algorithm. This particular study provides strong evidence that clinical phenomes, e.g., patient symptoms and signs, can be defined and predicted by genomic profiling. In addition to the prediction of patient phenomes per se, it is possible to define strains of infected bacteria and predict the severity and drug sensitivity of infection by measuring the metabolome and genome of those pathogens. Monk et al. (Monk et al., 2016) identified strain-specific differences by measuring genome, phenome, and transcriptome and genome-scale modeling and could predict high-production phenomes by defining specific characteristics of each omics profiling, establishing functional characteristics of metabolism from strain-specific genome-scale models, and integrating omics-specific models. This evidences that there are potential solutions to predicting phenomes of complex environmental factors and risks and better understanding the interaction between the environment and humans, between diseases and pathogens, and between phenomes and functions. It will be even more exciting if molecular omics profiling of the patient can be predicted from clinical phenomes, which provides the perfect figure of molecular medicine, although a large number of challenges have to be considered consciously (Hall et al., 2016).

\section{How far are we from clinical trans-omics for patients?}

Clinical trans-omics will be a systemic and comprehensive discipline to screen molecular multiomics according to pathological and clinical categories, define the connection and interaction between patient phenomes and molecules, and engineer multiomics networks of core elements from each individual network. This represents a new and high level of integrative biomedicine to explain the mechanism, early diagnosis, and precision medicine based on the interdisciplinary characteristics of transomics. However, there is an urgent need to develop new systems to create the matched individual networks of metabolomics, lipidomics, and clinical phenomics, and to integrate multiomics data networks in one repository for valuable, accessible, linkable, comprehensive, and dynamically updated and mined information. There is a need for comprehensive, public, and safe databases for clinical trans-omics data in hospitals where they also need a clinical bioinformatics and computational category of 
scientists to operate and maintain that information. We should ask if hospital and clinics are ready to handle such large-scale biomedicine and clinical trans-omics. A new step forward for clinical trans-omics is to regularly educate, train, and refresh multidisciplinary scientists and physicians. With the rapidly increased number of the "-omics," it is questioned whether the analytic methodology can be developed fast enough to define and integrate such large-scale trans-omics and how much of the trans-omics information can be utilized in clinical practice. In addition to the large bio-variance of molecular multiomics, the even larger variation of clinical phenomes should be seriously considered, although such variation will be narrowed down by more and more data generated from multiomics studies.

In conclusion, clinical trans-omics, different from multiomics, is a new concept and emerging science to integrate data of molecular multiomics, e.g., genomics, proteomics, metabolomics, and lipidomics, with clinical phenomics to identify diagnostic biomarkers and therapeutic targets. Clinical trans-omics emphasizes the valuable messages through clinical phenomes and molecular profiles, rather than the measurement of multiple omics simultaneously. Molecular multiomics describes the networks and correlations among genes, proteins, and lipids, while clinical trans-omics will provide the full picture of patient phenome-based molecular networks for clinical practice. The achievement and impact of clinical trans-omics will be to characterize patients, uncover mechanisms underlying drug responsiveness, and identify new disease-specific biomarkers and targets. Clinicians can easily link clinical phenomes with associated alternations of molecules, although there are still a number of challenges to be overcome.

\section{References}

Angione C, Conway M, Lió P. Multiplex methods provide effective integration of multi-omic data in genome-scale models. BMC Bioinformatics. 2016;17(Suppl 4):83. https://doi. org/10.1186/s12859-016-0912-1.

Chen C, Shi L, Li Y, Wang X, Yang S. Disease-specific dynamic biomarkers selected by integrating inflammatory mediators with clinical informatics in ARDS patients with severe pneumonia. Cell BiolToxicol. 2016;32(3):169-84. https://doi. org/10.1007/s10565-016-9322-4.

Chen H, Song ZJ, Qian MJ, Bai CX, Wang XD. Selection of disease-specific biomarkers by integrating inflammatory mediators with clinical informatics in AECOPD patients: a preliminary study. J Cell Mol Med. 2012a;16(6):1286-97.
Chen H, Wang YL, Bai CX, Wang XD. Alterations of serum inflammatory biomarkers in the healthy and chronic obstructive pulmonary disease patients with or without acute exacerbation. J Cell Mol Med. 2012b;16(6):1286-97.

Hall MA, Moore JH, Ritchie MD. Embracing complex associations in common traits: critical considerations for precision medicine. Trends Genet. 2016;32(8):470-84. https://doi. org/10.1016/j.tig.2016.06.001.

Kawamura Y, Takouda J, Yoshimoto K, Nakashima K. New aspects of glioblastoma multiforme revealed by similarities between neural and glioblastoma stem cells.Cell BiolToxicol. 2018. doi: https://doi.org/10.1007/s10565-017-9420-y.

Konno M, Matsui H, Koseki J, et al. Computational trans-omics approach characterisedmethylomic and transcriptomic involvements and identified novel therapeutic targets for chemoresistance in gastrointestinal cancer stem cells. Sci Rep. 2018;8(1):899. https://doi.org/10.1038/s41598-018-19284-3.

Lippert C, Sabatini R, Maher MC, Kang EY, Lee S, Arikan $\mathrm{O}$, et al. Identification of individuals by trait prediction using whole-genome sequencing data. Proc Natl AcadSci U S A. 2017;114(38):10166-71. https://doi. org/10.1073/pnas.1711125114.

Monk JM, Koza A, Campodonico MA, Machado D, Seoane JM, Palsson BO, et al. Multi-omics quantification of species variation of Escherichia coli links molecular features with strain phenotypes. Cell Syst. 2016;3(3):238-251.e12. https://doi.org/10.1016/j.cels.2016.08.013.

Shi L, Zhu B, Xu M, Wang X. Selection of AECOPD-specific immunomodulatory biomarkers by integrating genomics and proteomics with clinical informatics. Cell BiolToxicol. 2018;34(2):109-23. https://doi.org/10.1007/s10565-0179405-x.

Srivastava A, Kulkarni C, Mallick P, Huang K, Machiraju R. Building trans-omics evidence: using imaging and 'omics' to characterize cancer profiles. Pac SympBiocomput. 2018;23:377-87.

Sun YV, Hu YJ. Integrative analysis of multi-omics data for discovery and functional studies of complex human diseases. Adv Genet. 2016;93:147-90. https://doi.org/10.1016/bs. adgen.2015.11.004.

Wang X. New biomarkers and therapeutics can be discovered during COPD-lung cancer transition. Cell BiolToxicol. 2016;32(5):359-61. https://doi.org/10.1007/s10565-0169350-0.

Wang W, Wang X. Single-cell CRISPR screening in drug resistance. Cell BiolToxicol. 2017;33(3):207-10. https://doi. org/10.1007/s10565-017-9396-7.

Wang W, Zhu B, Wang X. Dynamic phenotypes: illustrating a single-cell odyssey. Cell BiolToxicol. 2017;33(5):423-7. https://doi.org/10.1007/s10565-017-9400-2.

Wu X, Sun X, Chen C, Bai C, Wang XD. Dynamic gene expressions of peripheral blood mononuclear cells in patients with acute exacerbation of chronic obstructive pulmonary disease: a preliminary study. Crit Care. 2014;18(6):508.

Wu D, Wang X, Sun H. The role of mitochondria in cellular toxicity as a potential drug target. Cell BiolToxicol. 2018;34(2):87-91. https://doi.org/10.1007/s10565-018-9425-1.

Yu X, Zeng T, Wang X, Li G, Chen L. Unravelling personalized dysfunctional gene network of complex diseases based on differential network model. J Transl Med. 2015;13:189. https://doi.org/10.1186/s12967-015-0546-5. 